\title{
THE POP-SWITCH PLANAR ALGEBRA AND THE JONES-WENZL IDEMPOTENTS
}

\author{
ELLIE GRANO AND STEPHEN BIGELOW
}

\begin{abstract}
The Jones-Wenzl idempotents are elements of the Temperley-Lieb planar algebra that are important, but complicated to write down. We will present a new planar algebra, the pop-switch planar algebra, which contains the Temperley-Lieb planar algebra. It is motivated by Jones' idea of the graph planar algebra of type $A_{n}$. In the tensor category of idempotents of the popswitch planar algebra, the $n$th Jones-Wenzl idempotent is isomorphic to a direct sum of $n+1$ diagrams consisting of only vertical strands.
\end{abstract}

\section{INTRODUCTION}

The Temperley-Lieb algebras were first introduced by Temperley and Lieb [LT71] in their work on transfer matrices in statistical mechanics. Vaughn F. R. Jones independently rediscovered Temperley-Lieb algebras in his work on von Neumann algebras [Jon99. He assembled these algebras together to form the Temperley-Lieb planar algebra, the simplest example of a subfactor planar algebra.

The Jones-Wenzl idempotents, first introduced in Wen87, are elements of the Temperley-Lieb algebras. One way they arise naturally is in representation theory. The Temperley-Lieb algebras encode the category of representations of $U_{q}\left(\mathfrak{s l}_{2}\right)$, and the Jones-Wenzl idempotents represent the irreducible representations. Chapters in books have been devoted to them [KL94]. They have been categorified by [CK10] and [FSS10, and generalized OY97].

While important, the Jones-Wenzl idempotents are difficult to write down explicitly. The $n$th Jones-Wenzl idempotent is a linear combination of every diagram with $n$ non-intersecting strands. The number of these diagrams is the $n$th Catalan number. To find the coefficient of a given diagram requires a complicated algorithm originally given by Frankel and Khovanov [FK97] and later written down by Morrison Mor.

In this paper, we define the pop-switch planar algebra, a new planar algebra that contains the Temperley-Lieb planar algebra. Our original motivation was a diagrammatic treatment of the graph planar algebra introduced by Jones [Jon00. The pop-switch planar algebra captures with simple diagrams the complicated calculations involved in working with objects in the graph planar algebra.

The main theorem of this paper shows that each Jones-Wenzl idempotent is isomorphic to a direct sum of diagrams with only vertical strands. It is to be hoped that this makes them easier to work with, and gives a new approach to some open problems. 


\section{BACKGROUND}

For convenience, we work over the field $\mathbb{C}$ and let $q$ be a nonzero complex number that is not a root of unity. Many of the results hold over other fields, but if $q$ is a root of unity the proofs fail due to division by zero.

Definition 2.1. The $n$th quantum number is defined as

$$
[n]=[n]_{q}=\frac{q^{n}-q^{-n}}{q-q^{-1}}
$$

and the quantum binomial is defined as

$$
\left[\begin{array}{l}
n \\
k
\end{array}\right]=\frac{[n][n-1] \cdots[n-k+1]}{[k][k-1] \cdots[1]}
$$

where $0 \leq k \leq n$ are natural numbers.

We have the following identities.

Lemma 2.2. $[k+l]=[k][l+1]-[k-1][l]$.

Proof. This follows from the definition and a simple computation.

Corollary 2.3. $\left[\begin{array}{c}k+l \\ l\end{array}\right]=[l+1]\left[\begin{array}{c}k+l-1 \\ l\end{array}\right]-[k-1]\left[\begin{array}{c}k+l-1 \\ l-1\end{array}\right]$.

Proof. After taking a common denominator and cancelling common terms, this reduces to the previous lemma.

2.1. Planar algebras. We won't define planar algebras in great detail. See Jones' original paper [Jon99] for a formal definition. See MPS08] for a helpful introduction.

We will use what are sometimes called vanilla planar algebras. These lack any of the optional extra features or properties that are often included in the definition.

A planar tangle $T$ consists of:

- a disk $D$ called the output disk,

- a finite set of disjoint disks $D_{i}$ called the input disks in the interior of $D$,

- a point called a basepoint of $\partial D$ and of each $\partial D_{i}$, and

- a collection of disjoint curves called strands in $D$.

The strands can be closed curves, or can have endpoints on $\partial D$ or $\partial D_{i}$ or both. Apart from the endpoints, the strands lie in the interior of $D$ and do not intersect $D_{i}$. The basepoints do not coincide with endpoints of strands. Planar tangles are considered up to isotopy in the plane.

It is sometimes possible to insert a planar tangle $T_{1}$ into one of the input disks of another planar tangle $T_{2}$ to obtain a new planar tangle. Specifically, this is possible if the number of endpoints on the output disk of $T_{1}$ is the same as the number of endpoints on the chosen input disk of $T_{2}$. Then we can use an isotopy to make the endpoints match up. This still leaves an ambiguity of how to rotate $T_{1}$. The basepoints remove this ambiguity: we require the basepoint of the output disk of $T_{1}$ to coincide with the basepoint of the chosen input disk of $T_{2}$.

The planar tangles, together with this operation of inserting one planar tangle into an input disk of another, form a rather general type of algebraic gadget called an operad. Briefly, a planar algebra is a representation of the operad of planar tangles. 
More concretely, a planar algebra $\mathcal{P}$ is a sequence of vector spaces $\mathcal{P}_{i}$ for $i \geq 0$. Suppose $T$ is a planar tangle with input disks $D_{1}, \ldots, D_{n}$. Let $d_{i}$ be the number of endpoints on $\partial D_{i}$ and let $d$ be the number of endpoints on $\partial D$. Suppose $v_{i} \in \mathcal{P}_{d_{i}}$ for all $i$. Then there is an action of $T$

$$
T\left(v_{1}, \ldots, v_{n}\right) \in \mathcal{P}_{d} .
$$

The action of planar tangles must be multilinear, and it must be compatible with the operad structure in a natural sense.

The definition of a planar algebra may seem complicated. However it formalizes a fairly simple idea, familiar to knot theorists, of tangle-like diagrams that can be glued together in arbitrary planar ways. Perhaps the main novelty is that we allow formal linear combinations of diagrams, which glue together in a multilinear way.

An example might help.

2.2. The Temperley-Lieb planar algebra. The simplest planar algebra is the Temperley-Lieb planar algebra $\mathcal{T} \mathcal{L}$. The vector space $\mathcal{T} \mathcal{L}_{i}$ is spanned by tangle diagrams that have no input disks and $i$ endpoints on the output disk.

There is one relation. A closed loop in a diagram may be deleted at the expense of multiplying by the scalar $q+q^{-1}$. We call this the bubble-bursting relation.

If $i$ is odd then $\mathcal{T}_{i}$ is zero. A basis for $\mathcal{T}_{2 n}$ is given by tangle diagrams that have $n$ strands and no closed loops.

In practice, most planar algebras can be thought of as formal linear combinations of diagrams that are similar to Temperley-Lieb diagrams, but with optional extra features, like crossings, orientations, colors, or vertices.

2.3. The category corresponding to a planar algebra. Suppose $\mathcal{P}$ is a planar algebra. We now describe how $\mathcal{P}$ can be thought of as a category. In this context, the input and output disks in the definition of $\mathcal{P}$ should be thought of as rectangles instead of round disks.

The category $\mathbf{C}$ corresponding to $\mathcal{P}$ is as follows.

- The objects are the non-negative integers.

- The morphisms from $i$ to $j$ are the elements of $\mathcal{P}_{i+j}$, thought of as having $i$ endpoints on the bottom of the rectangle and $j$ on the top.

- The composition $f \circ g$ is given by stacking $f$ on top of $g$.

Let $\mathcal{P}_{i}^{j}$ denote $\mathcal{P}_{i+j}$ with the elements treated as morphisms from $i$ to $j$.

An idempotent is an element $p$ of $\mathcal{P}_{n}^{n}$ such that $p^{2}=p$.

We can expand the objects in the category by a construction known as the Karoubi envelope. This new category $\mathbf{C}^{\prime}$ is defined as follows.

- The objects of $\mathbf{C}^{\prime}$ are the idempotents of $\mathbf{C}$.

- The morphisms from $p$ to $q$ are morphisms in $\mathbf{C}$ of the form $q x p$.

Next, note that $\mathbf{C}$ and $\mathbf{C}^{\prime}$ are also tensor categories, where $x \otimes y$ is obtained by placing $x$ to the left of $y$.

Finally, we can define a matrix category of $\mathbf{C}^{\prime}$. The objects are formal direct sums of objects of $\mathbf{C}^{\prime}$ and the morphisms are formal matrices. Instead of this abstract definition, all we need is the following lemma.

Lemma 2.4. Suppose $p$ and $q_{1}, \ldots, q_{n}$ are idempotents such that

$$
p=q_{1}+\cdots+q_{n},
$$


and $q_{i} q_{j}=0$ whenever $i \neq j$. Then

$$
p \simeq q_{1} \oplus \cdots \oplus q_{n} .
$$

2.4. Jones-Wenzl idempotents. The Jones-Wenzl idempotent $p_{n}$ is the unique element of $\mathcal{T} \mathcal{L}_{n}^{n}$ such that

- $p_{n} \neq 0$

- $p_{n}^{2}=p_{n}$

- $a p_{n}=0$ if $a$ is any diagram that includes a strand with both endpoints at the bottom of the rectangle.

- $p_{n} b=0$ if $b$ is any diagram that includes a strand with both endpoints at the top of the rectangle.

Because of these last two properties, the Jones-Wenzl idempotents are sometimes referred to as "uncappable." If $q$ is a root of unity, the Jones-Wenzl idempotents do not exist for all $n$.

\section{THE POP-SWITCH PLANAR ALGEBRA}

\subsection{The pop-switch planar algebra.}

Definition 3.1. Let the pop-switch planar algebra $\mathcal{P S P} \mathcal{A}$ be the planar algebra generated by oriented strands modulo the following relations.

- The pop-switch relations

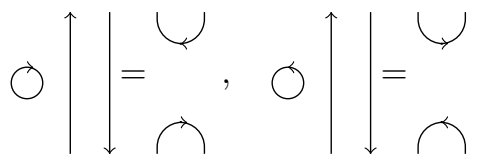

- The bubble-bursting relation

$$
\bigcirc+\bigcirc=\left(q+q^{-1}\right) \epsilon
$$

where $\epsilon$ denotes the empty diagram.

This contains the Temperley-Lieb planar algebra; a non-oriented strand is the sum of each orientation.

We need some tools to move the diagrams around.

Denote $n$ parallel strands oriented in the same direction by a single oriented strand labelled $n$.

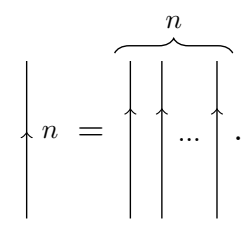

If $n$ is a negative integer, ${ }^{n}=\left.\right|_{-n}$

Let $\iota_{n}$ denote $n$ vertical strands oriented up. Let $\beta_{n}$ denote $n$ parallel strands that form a bubble oriented counterclockwise. Let $\alpha_{n}$ denote a $\beta_{-n}$ inside a $\beta_{n}$.

$$
\iota_{n}=\uparrow^{n} \quad \beta_{n}=\bigcirc^{n} \quad \alpha_{n}=\bigcirc^{n}{ }^{n} .
$$

Lemma 3.2. Suppose $x \in \mathcal{P S P} \mathcal{A}_{0}$ and $y$ is a sequence of $2 n$ vertical strands such that $n$ are oriented up and $n$ are oriented down. Then $x \otimes y=y \otimes x$. 
Proof. Use the pop-switch relation repeatedly to create a gap and pass $x$ through. Then use the pop-switch relation repeatedly to restore the original $2 n$ vertical strands.

Lemma 3.3. The multi-pop-switch relations The pop-switch relations hold for multiple strands.

$$
\left.\bigcirc^{n}\right|_{n} ^{n}=\overbrace{n}^{n},\left.\left.\quad \bigcirc^{n}\right|_{n}\right|_{n}=\mho_{n}^{n}
$$

Proof. Without loss of generality, consider the first equality. Induct on $n$. The case $n=1$ is the pop-switch relations. For the case $n=k+1$, move the innermost $\beta_{-k}$ across two strands using the previous lemma. Then use the case $n=k$, and finally the case $n=1$.

Corollary 3.4. $\iota_{k} \otimes \alpha_{n}=\iota_{k}$ and $\iota_{-k} \otimes \alpha_{-n}=\iota_{-k}$ for $k \geq n \geq 0$.

Proof. Consider $\iota_{k} \otimes \alpha_{n}$. Use the multi-pop-switch relation by popping the innermost $\beta_{n}$ of the $\alpha_{n}$. Then straighten out the $\iota_{n}$. The other case is similar.

\section{Corollary 3.5.}

$$
\bigcirc)^{n}=\alpha_{n} \otimes \beta_{n-1} \quad \text { and } \bigcirc{ }^{n}=\alpha_{-n} \otimes \beta_{-n+1} \text {. }
$$

Proof. Start with the left side of the first equality. Use a multi-pop-switch relation on the $n-1$ strands, as shown below.

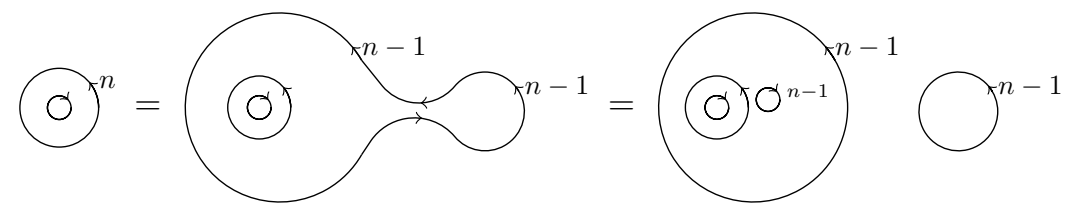

By Lemma 3.2 we can move the $\beta_{-n+1}$ into the $\alpha_{1}$ to achieve the result.

$$
=\bigcirc^{n} \bigcirc^{n-1}=\alpha_{n} \otimes \beta_{n-1}
$$

The second identity is proved similarly.

Lemma 3.6. $\iota_{n}=\beta_{-n} \otimes \iota_{n} \otimes \beta_{n}$.

Proof. This follows from the multi-pop switch relations.

$$
\iota_{n}=\uparrow^{n}=\Upsilon^{n}=\sigma^{n} \uparrow^{n} \bigcirc^{n}=\beta_{-n} \otimes \iota_{n} \otimes \beta_{n}
$$

Now we give some relations involving the Jones-Wenzl idempotents $p_{n}$. First, we need some notation for them. We will use a rectangle to represent $p_{n}$. It should always be assumed that $p_{n} \in P_{n}^{n}$ even if the strands are not drawn.

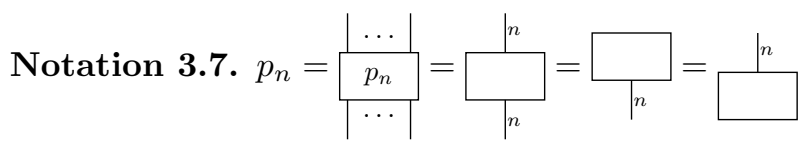


We can make use of the the fact that they are uncappable.

Lemma 3.8. ${ }_{\uparrow} \circlearrowright=(-1)^{n+1} \bigcup_{{ }_{n}} \bigcirc^{n}$ This relation remains true if all arrows are reversed.

Proof. For the case $n=0$, use the fact that an unoriented cap gives zero. For the case $n=1$, use the case $n=0$ and the pop-switch relation.

For the general case, use induction on $n$. Start by using the case $n=k$ as follows:

$$
\prod_{k+1}=\prod_{\uparrow k} \circlearrowright=(-1)^{k+1} \Upsilon_{\uparrow_{k}} \bigcirc^{k}
$$

Next use the case $n=1$, followed by Lemma 3.2 , to achieve the result.

$$
\begin{aligned}
& =(-1)^{k+2} \bigvee_{{ }_{k}} \bigcirc^{k}=(-1)^{k+2} \bigvee_{\sigma_{\uparrow}} \bigcirc^{k} \\
& =(-1)^{k+2} \circlearrowright \bigcirc_{k+1} \bigcirc^{k+1}
\end{aligned}
$$

\section{Proof of the main theorem}

The aim of this section is to prove the following.

Theorem 4.1. The nth Jones-Wenzl idempotent is isomorphic to a direct sum of $n+1$ diagrams:

$$
\begin{aligned}
p_{n} & \simeq \bigoplus_{i=0}^{n} \iota_{-i} \otimes \iota_{n} \otimes \iota_{-i} \\
& =\left.\left.\uparrow^{n} \oplus\left|\uparrow^{n} \downarrow \oplus \cdots \oplus\right|\right|_{n}\right|^{n} \downarrow_{n}
\end{aligned}
$$

Proof. Since $p_{n}$ is an idempotent, $p_{n}=p_{n}^{2}=p_{n} \operatorname{id}_{n} p_{n}$, where $\mathrm{id}_{n}$ is $n$ nonoriented parallel strands, the multiplicative identity in $\mathcal{T} \mathcal{L}_{n}^{n}$. Now write $\mathrm{id}_{n}$ as a sum of $2^{n}$ different ways of orienting $n$ vertical strands. Break this sum into $n+1$ sums depending on how many strands are oriented up.

Definition 4.2. Let $p_{n-k}^{k}$ denote the sum of $\left(\begin{array}{l}n \\ k\end{array}\right)$ diagrams obtained from $p_{n} \operatorname{id}_{n} p_{n}$ by orienting $k$ strands up and $n-k$ strands down in the $\operatorname{id}_{n}$.

Then $p_{n}=p_{n}^{0}+p_{n-1}^{1}+\cdots+p_{0}^{n}$. If $k_{1} \neq k_{2}$, then $p_{n-k_{1}}^{k_{1}} p_{n-k_{2}}^{k_{2}}=0$. Thus, by Lemma 2.4 .

It remains only to show

$$
p_{n} \simeq p_{n}^{0} \oplus p_{n-1}^{1} \oplus \cdots \oplus p_{0}^{n} .
$$

This is done in Lemma 4.9

$$
p_{l}^{k} \simeq \iota_{-l} \otimes \iota_{k+l} \otimes \iota_{-l}
$$

To prove Lemma 4.9 , we first define $X_{l}^{k}$, which we will show is equal a scalar times $p_{l}^{k}$ in Lemma 4.8 . 


\section{Definition 4.3.}

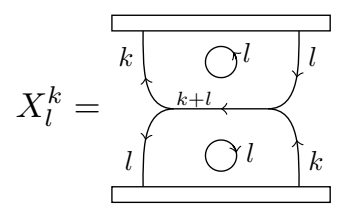

Lemmas 4.4 and 4.5 are similar and begin the inductive step of the proof of Lemma 4.8

Lemma 4.4. $p_{k+l}\left(X_{l}^{k-1} \otimes \iota_{1}\right) p_{k+l}=(-1)^{l} X_{l}^{k} \otimes \beta_{-l}$.

Proof.

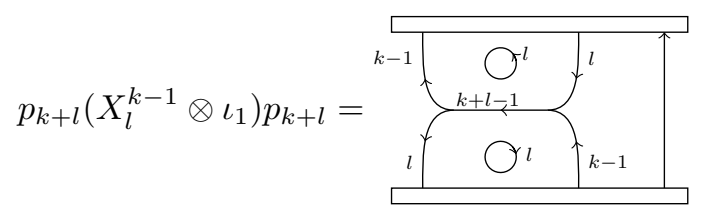

By a pop-switch relation we have the following.

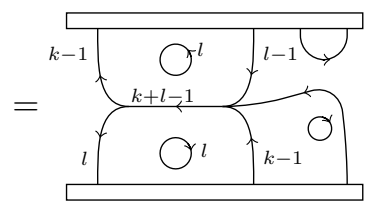

Then by Lemma 3.8 we can move the arc across the $l-1$ strands creating a $\beta_{l-1}$ on the right. Next we use Lemma 3.6 to replace the arc with $\beta_{-1} \otimes \iota_{1} \otimes \beta_{1}$.

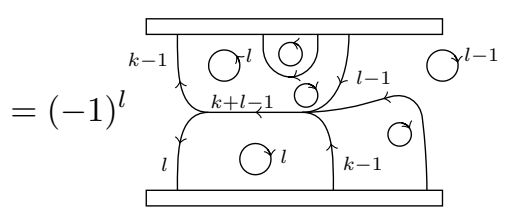

Move the innermost $\beta_{1}$ from the $\beta_{l}$ to the far right across $l-1$ strands in both directions by Lemma 3.2 .

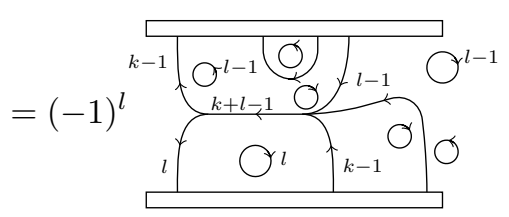


Then remove the two bubbles on the bottom right of the diagram by Lemma 3.6.

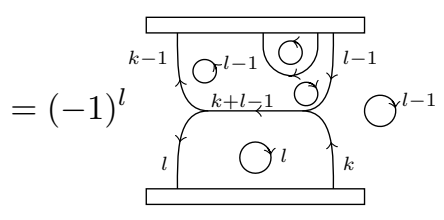

Lastly, by Lemma 3.2 move the $\beta_{l-1}$ into the $\beta_{1}$ and the $\beta_{-1}$ into the $\beta_{-(l-1)}$.

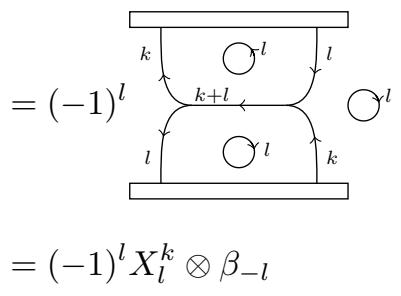

Lemma 4.5. $p_{k+l}\left(X_{l-1}^{k} \otimes \iota_{-1}\right) p_{k+l}=(-1)^{k} X_{l}^{k} \otimes \beta_{k}$.

Proof.

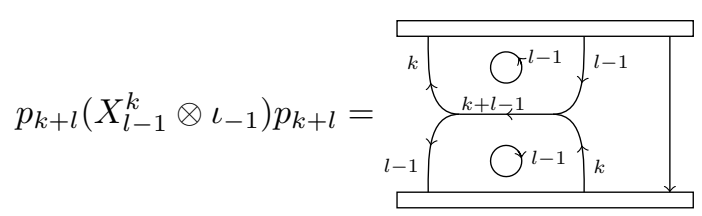

By a pop-switch relation we have the following.

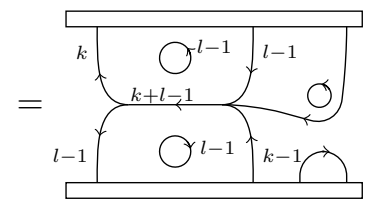

Then by Lemma 3.8 we can move the arc across the $k-1$ strands creating a $\beta_{-}(k-1)$ on the right. Next we use Lemma 3.2 to move the $\beta_{1}$ across the $l-1$ strands in both directions into the $\beta_{l-1}$.

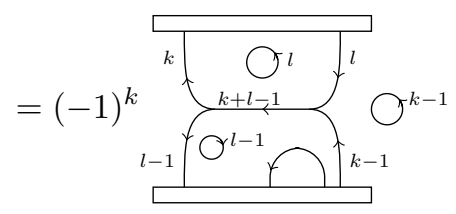


Replace the arc with $\beta_{-1} \otimes \iota_{1} \otimes \beta_{1}$.

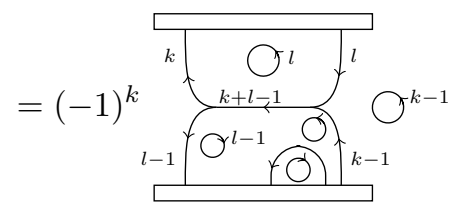

Lastly, by Lemma 3.2 move the $\beta_{1}$ across the $k-1$ strands in both directions into the $\beta_{k-1}$. By the same lemma, move the $\beta_{-(l-1)}$ into the $\beta_{-1}$.

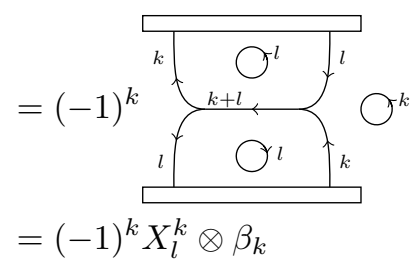

Lemma 4.6 is the key to proving Lemma 4.7. which is required to complete the proof of Lemma 4.8. It is worth noting that in Lemma 4.7 the $X_{l}^{k}$ merely acts as a catalyst to provide enough strands to use 4.6 All that is necessary is the presence of $\iota_{-l+1}$ and $\iota_{k-1}$ on the left as specified in Lemma 4.6 for the purpose of implementing Corollary 3.4 .

Lemma 4.6. For $k \geq n-1$,

$$
\iota_{k} \otimes \beta_{n}=[n] \iota_{k} \otimes \beta_{1}-[n-1] \iota_{k}
$$

and

$$
\iota_{-k} \otimes \beta_{-n}=[n] \iota_{-k} \otimes \beta_{-1}-[n-1] \iota_{-k}
$$

Proof. We prove the first identity, since the second is similar. Consider the case $n=2$ with $k \geq 1$. Use the bubble-bursting relation on the innermost loop of $\beta_{2}$. Corollary 3.4 then gives the result.

$$
\iota_{k} \otimes \beta_{2}=[2] \iota_{k} \otimes \beta_{1}-\iota_{k} \otimes \alpha_{1}=[2] \iota_{k} \otimes \beta_{1}-\iota_{k}
$$

Now assume $k \geq n-1$. Use the bubble-bursting relation on the innermost loop of $\beta_{n}$. Corollary 3.5. Corollary 3.4, and induction give

$$
\begin{aligned}
\iota_{k} \otimes \beta_{n} & =[2] \iota_{k} \otimes \beta_{n-1}-\iota_{k} \otimes \beta_{n-2} \\
& =[2]\left([n-1] \iota_{k} \otimes \beta_{1}-[n-2] \iota_{k}\right)-\left([n-2] \iota_{k} \otimes \beta_{1}-[n-3] \iota_{k}\right) \\
& =([2][n-1]-[n-2]) \iota_{k} \otimes \beta_{1}-([2][n-]-[n-3]) \iota_{k} \\
& =[n] \iota_{k} \otimes \beta_{1}-[n-1] \iota_{k}
\end{aligned}
$$


Lemma 4.7. If $k+l=n$ then

$$
\left[\begin{array}{c}
n-1 \\
l
\end{array}\right] X_{l}^{k} \otimes \beta_{-l}+\left[\begin{array}{c}
n-1 \\
k
\end{array}\right] X_{l}^{k} \otimes \beta_{k}=\left[\begin{array}{l}
n \\
k
\end{array}\right] X_{l}^{k} .
$$

Proof. Note that every term in the equation contains $X_{l}^{k}$. However, the result will hold so long as there are both a $\iota_{-l+1}$ and $\iota_{k-1}$ on the left of each diagram in order to use Lemma 4.6. Thus it suffices to prove

$$
\left[\begin{array}{c}
n-1 \\
l
\end{array}\right]\left([l] \beta_{-1}-[l-1]\right)+\left[\begin{array}{c}
n-1 \\
k
\end{array}\right]\left([k] \beta_{1}-[k-1]\right)=\left[\begin{array}{l}
n \\
k
\end{array}\right] .
$$

Use the identity

$$
\left[\begin{array}{c}
n-1 \\
l
\end{array}\right][l]=\left[\begin{array}{c}
n-1 \\
k
\end{array}\right][k],
$$

and the bubble bursting relation $\beta_{-1}+\beta_{1}=[2]$ to eliminate $\beta_{-1}$ and $\beta_{1}$ from the left side. Then simplify further using the identity $[2][l]-[l-1]=[l+1]$. We obtain

$$
\left[\begin{array}{c}
n-1 \\
l
\end{array}\right][l+1]-\left[\begin{array}{c}
n-1 \\
k
\end{array}\right][k-1] .
$$

By Corollary 2.3 this is equal to $\left[\begin{array}{l}n \\ k\end{array}\right]$, as desired.

Lemma 4.8. $p_{l}^{k}=(-1)^{k l}\left[\begin{array}{c}k+l \\ k\end{array}\right] X_{l}^{k}$

Proof. Induct on $n=k+l$. Notice $p_{0}^{1}=\iota_{1}=X_{0}^{1}$ and $p_{1}^{0}=\iota_{-1}=X_{1}^{0}$. Assume $k>0$ and $l>0$. Then

$$
p_{l}^{k}=p_{k+l}\left(p_{l}^{k-1} \otimes \iota_{1}\right) p_{k+l}+p_{k+l}\left(p_{l-1}^{k} \otimes \iota_{-1}\right) p_{k+l}
$$

By Lemma 4.4 and Lemma 4.5 .

$$
=(-1)^{k l}\left[\begin{array}{c}
k+l-1 \\
l
\end{array}\right] X_{l}^{k} \otimes \beta_{-l}+(-1)^{k l}\left[\begin{array}{c}
k+l-1 \\
k
\end{array}\right] X_{l}^{k} \otimes \beta_{k}
$$

By Lemma 4.7 .

$$
=(-1)^{k l}\left[\begin{array}{c}
k+l \\
k
\end{array}\right] X_{l}^{k}
$$

Lemma 4.9. $p_{l}^{k} \simeq \iota_{-l} \otimes \iota_{k+l} \otimes \iota_{-l}$.

Proof. The explicit isomorphisms are:

$$
f=(-1)^{k l}\left[\begin{array}{c}
k+l \\
k
\end{array}\right] \stackrel{h^{l}}{k} \mathrm{O}^{l} f_{l}, \quad g=\text { lf } O^{l} f_{k} .
$$

Then $f \circ g=(-1)^{k l}\left[\begin{array}{c}k+l \\ k\end{array}\right] X_{l}^{k}=p_{l}^{k}$ by Lemma 4.8 . Thus $f \circ g$ is the identity morphism from $p_{l}^{k}$ to $p_{l}^{k}$.

On the other hand, $g \circ f=\iota_{-l} \otimes \iota_{k+l} \otimes \iota_{-l}$, the identity morphism from $\iota_{-l} \otimes$ $\iota_{k+l} \otimes \iota_{-l}$ to $\iota_{-l} \otimes \iota_{k+l} \otimes \iota_{-l}$. 


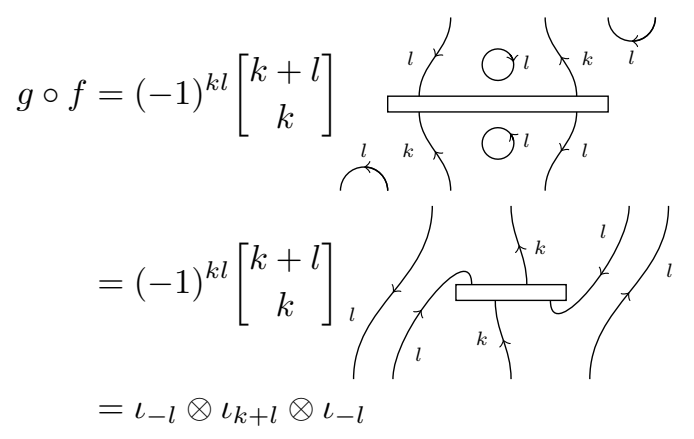

The second equality holds by performing two multi-pop-switch relations: one on the $\beta_{-l}$ at the top with the $l$ strands to the left and the $l$ strands on the bottom left, and the other on the $\beta_{l}$ and the $l$ strands on the right and top right. Now expand the Jones-Wenzl idempotent. The only non-zero term come from one of the following Temperley-Lieb diagrams.
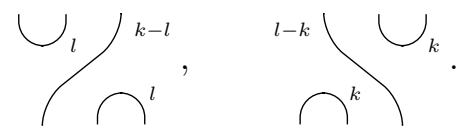

Thus the result of $g \circ f$ must be a scalar times $\iota_{-l} \otimes \iota_{k+l} \otimes \iota_{-l}$. Since $f \circ g$ is the identity and $g \circ f$ is a scalar times the identity, that scalar must be 1 .

\section{Graph Planar algebra and the Temperley-Lieb Planar algebra}

This section is motivation for the definition of the pop-switch planar algebra. We start with a summary of the definition of the graph planar algebra, first defined in [Jon00].

Throughout this section, fix a simple graph $\Gamma$. For Jones, all planar algebras are shaded, and $\Gamma$ is required to be bipartite. We will ignore this issue.

Let $\mu$ be a function from the vertices of $\Gamma$ to $\mathbf{R}_{>0}$. We will define the graph planar algebra $\mathcal{P}$ corresponding to $(\Gamma, \mu)$.

For each $k>0$, let $\mathcal{P}_{2 k}$ be the vector space of complex valued functions on the set of loops of length $2 k$ on $\Gamma$.

Suppose $T$ is a tangle. For each input disk of $T$, let $v_{b}$ be a corresponding input vector. We must define a corresponding output vector $v$. Thus we must define $v(\gamma)$ for every loop $\gamma$ in $\Gamma$ that has length equal to the number of endpoints on the outer boundary of $T$.

A state $\sigma$ of $T$ is a function from the set of regions of $T$ to the set of vertices of $\Gamma$ such that adjacent regions are sent to adjacent vertices.

Suppose $r$ is a region of $T$. This is a planar surface with boundary that may include some right-angled corners. The Euler measure $e(r)$ is defined in a similar way to the Euler characteristic, using the usual formula $V-E+F$ for a triangulation of $r$. The difference is, every corner must be a vertex and only counts as $\frac{1}{4}$, any other vertex on a boundary only counts as $\frac{1}{2}$, and every edge on a boundary only counts as $\frac{1}{2}$. 
We are finally ready to define the image vector $v$ of the vectors $v_{b}$ under the action of the tangle $T$.

$$
v(\gamma)=\sum_{\sigma}\left(\prod_{r} \mu(\sigma(r))^{e(r)}\right)\left(\prod_{b} v_{b}\left(\left.\sigma\right|_{\partial b}\right)\right) .
$$

The sum is over all states $\sigma$ that are compatible with $\gamma$. The first product is over all regions $r$ of $T$. The second product is over all input disks $b$ of $T$.

The Temperley-Lieb planar algebra is a subfactor planar algebra of type $A_{\infty}$. It can be found inside the graph planar algebra associated to $\Gamma=A_{\infty}$, which is the ray with vertices indexed by positive integers. The function $\mu$ assigns the quantum integer $[n]$ to the $n$th vertex. (Note we are still assuming $q$ is not a root of unity. If $q$ is a primitive $(n+1)$ th root of unity then we should use the graph $A_{n}$.)

Suppose $T$ is an oriented tangle. Define a state of $T$ to be a function from the set of regions of $T$ to the set of vertices of $A_{\infty}$ such that, for any strand of $T$, if the region to its right is sent to vertex $n$ then the region to its left is sent to vertex $n+1$. Thus, a state is determined by the vertex associated to a single region. In a sense, the orientation on the strands removes the ambiguity in the state of a Temperley-Lieb diagram.

Now suppose $T$ and $T^{\prime}$ differ by a pop-switch relation. There is an obvious correspondence between states of $T$ and states off $T^{\prime}$. Furthermore, the total Euler measure of the region associated to any given vertex is the same. We therefore have a well-defined embedding of the pop-switch planar algebra in the graph planar algebra of the graph $A_{\infty}$.

One can think of the pop-switch planar algebra as a diagrammatic way to keep track of computations inside the graph planar algebra of $A_{\infty}$.

\section{REFERENCES}

[CK10] B. Cooper and V. Krushkal. Categorification of the Jones-Wenzl projectors. ArXiv eprints, May 2010.

[FK97] I. Frenkel and M. Khovanov. Canonical bases in tensor products and graphical calculus for $u_{q}\left(\mathfrak{s l}_{2}\right)$. Duke Math. J., 87(3):409-480, 041997.

[FSS10] I. Frenkel, C. Stroppel, and J. Sussan. Categorifying fractional euler characteristics, Jones-Wenzl projector and $\$ 3$ j\$-symbols. ArXiv e-prints, July 2010.

[Jon99] V. F. R. Jones. Planar algebras, I. ArXiv Mathematics e-prints, September 1999.

[Jon00] V. F. R. Jones. The planar algebra of a bipartite graph. Knots in Hellas '98, 24:94-117, 2000.

[KL94] L. Kauffman and S. Lins. Temperley-Lieb Recoupling Theory and Invariants of 3Manifolds. Princeton University Press, 1994.

[LT71] H. N. V. Lieb and E. H. Temperley. Relations between the 'Percolation' and 'Colouring' Problem and other Graph-Theoretical Problems Associated with Regular Planar Lattices: Some Exact Results for the 'Percolation' Problem. Proceedings of the Royal Society of London., pages 251-280, April 1971.

[Mor] S. Morrison. A formula for the Jones-Wenzl projections. Unpublished. Available at https://tqft.net/math/JonesWenzlProjections.pdf.

[MPS08] S. Morrison, E. Peters, and N. Snyder. Skein theory for the $\mathrm{d}_{2 n}$ planar algebras. ArXiv e-prints, August 2008.

[OY97] T. Ohtsuki and S. Yamada. Quantum su(3) invariant of 3-manifolds via linear skein theory. Journal of Knot Theory and Its Ramifications, 06(03):373-404, 1997.

[Wen87] H. Wenzl. On sequences of projections. C. R. Math. Rep. Acad. Sci. Canada, 9(1):5-9, 1987. 
THE POP-SWITCH PLANAR ALGEBRA AND THE JONES-WENZL IDEMPOTENTS

Ellie Grano, Pepperdine University-NASC, 24255 Pacific Coast Hwy, Malibu, CA 90263-4321

E-mail address: ellie.grano@pepperdine.edu

Stephen Bigelow, Dept of Math, South Hall Room 6607, University of California, Santa Barbara, CA93106

E-mail address: bigelow@math.ucsb.edu 